University of Tennessee Health Science Center UTHSC Digital Commons

12-5-2019

\title{
Sepsis Screening Tool Increased the Usage of Sepsis Order Set
}

Raweewan Liengsawangwong

University of Tennessee Health Science Center

Follow this and additional works at: https://dc.uthsc.edu/hiimappliedresearch

Part of the Health and Medical Administration Commons, and the Health Information Technology Commons

\section{Recommended Citation}

Liengsawangwong, Raweewan, "Sepsis Screening Tool Increased the Usage of Sepsis Order Set" (2019). Applied Research Projects. 67. . https://doi.org/10.21007/chp.hiim.0066

https://dc.uthsc.edu/hiimappliedresearch/67

This Research Project is brought to you for free and open access by the Department of Health Informatics and Information Management at UTHSC Digital Commons. It has been accepted for inclusion in Applied Research Projects by an authorized administrator of UTHSC Digital Commons. For more information, please contact jwelch30@uthsc.edu. 
Sepsis screening tool increased the usage of sepsis order set

Raweewan Liengsawangwong

MHIM 613

Associate Professor Sajeesh Kumar, Ph. D.

The University of Tennessee Health Science Center

The University of Tennessee Institutional Review Board: 18-05810-NHRS. 
Table of content

Chapter 1 Introduction and background

Chapter 2 Literature review

Chapter 3 Materials and methods

Chapter 4 Research results 30

Chapter 5 Discussion and Analysis

33

Chapter 6 Discussion

36

Bibliography 


\author{
Abstract \\ Sepsis screening tool increased the usage of sepsis order set \\ Raweewan Liengsawangwong, $\mathrm{MD}^{1}$; Sajeesh Kumar, $\mathrm{PhD}^{2}$; Ruben A. Ortiz ; Jason Hill, MD, \\ $\mathrm{MSc}^{4}$ \\ ${ }^{1}$ Department of Health Informatics and Information Management, The University of Tennessee Health Science Center, Memphis, TN. \\ ${ }^{2}$ Department of Health Informatics and Information Management, The University of Tennessee Health Science Center, Memphis, TN. \\ ${ }^{3}$ Office of Information Technology, The University of Tennessee, Knoxville, TN. \\ ${ }^{4}$ Medical Director of Informatics, Ochsner Medical Center Northshore, LA. \\ ${ }^{5}$ Section Head of OMC Hospital Medicine-Northshore and Associated CMIO, Ochsner Health System, LA \\ Acknowledgment: Thank you, Ms. Marlene Alonzo, MSN, RN-BC ${ }^{5}$ for providing the data
}

Introduction: The sepsis screening tool was launched to improve the usage of the sepsis order set.

Objectives: The purposes of this study were to determine whether the sepsis screening tool increased the usage of sepsis order set and whether the tool improved the primary outcomes. This study assessed the association between using sepsis order set and the compliance of the SEP-1 measure (represented by achieving total perfect care), and the primary outcomes. Furthermore, this study assessed association between the compliance with SEP-1 and the primary outcomes. The primary outcomes were a) time zero to antibiotics, b) inpatient length of stay, and c) survival at discharge.

Material and Methods: Retrospective study collected data 6 months before and 6 months after the launch of sepsis screening tool at a tertiary academic hospital. A total of 632 patients were studied. The sepsis screening tool was incorporated at the nurse station at triage. The sepsis order set contained treatment guidelines based on the SEP-1 measure.

Results: Our findings confirmed that the sepsis screening tool increased the usage of the order set, raised an awareness of the emergency department personnel and improved the adherence to 
the treatment guidelines by showing that the usage of sepsis order set significantly increased in the postintervention group $(p=0.001)$. However, we did not find the association between the sepsis screening tool and the primary outcomes or the total perfect care. The utilization of sepsis order set streamlined and standardized the sepsis management, shortened time to antibiotic by 54 minutes $(p=0.001)$ and reduced length of stay by 1.8 days $(p=0.002)$. However, there was no significant difference in survival between the group that used the order set and the group that did not use the order set. There was a significant association between sepsis order set use and total perfect care $(p<0.001)$, which indicated that the order set use increased the compliance with SEP-1 measure. The group that achieved total perfect care significantly associated with all primary outcomes; 102.4 minutes shorter average time zero to antibiotic $(p<0.001), 1.5$ days shorter average length of stay $(p=0.004)$, and better survival at discharge $(p<0.001,95 \%$ CI $0.02-0.206$, OR 0.064$)$ than the group that did not achieve total perfect care.

Conclusions: Our study confirmed that adherence to the standard treatment guidelines improved the treatment outcomes. The sepsis screening tool increased the use of the sepsis order set. When the order set was used, the compliance with the SEP-1 measure increased. The group that used sepsis order set had a significantly shorter length of stay and shorter time to antibiotic. The group that met SEP-1 measure compliance significantly received antibiotics earlier, shorter stay as an inpatient, and better survival. However, we need more studies to confirm the significant association between compliance of SEP-1 measure and the outcomes because this study did not adjust for clinical characteristics and severity of illness. 


\section{Chapter 1 Introduction and background}

Sepsis and septic shock are major healthcare problems, affecting millions of people around the world each year, and killing as many as one in four (and often more) (Rhodes et al., 2017). The treatment of sepsis is complicated and costly. The Healthcare Cost and Utilization Project (HCUP) reported that in 2013, septicemia was the most expensive condition treated, accounting for $\$ 23.7$ billion, or 6.2 percent of the aggregate costs for all hospitalizations (Torio \& Moore, 2016). The burden of expensive health care expenditures takes a significant share of the Gross Domestic Product and creates a burden on governments, consumers, and insurers. Even with the comprehensive treatment, the mortality of ICU patients with severe sepsis or septic shock is as high as 30-40\% (Nesseler et al., 2013). The delayed treatment of sepsis can rapidly advance to septic shock, multiple organ dysfunction syndromes (MODS), and death (Amland \& Hahn-Cover, 2019). The prompt management of sepsis would yield a reduction in the risk of complication, organ failure, and mortality.

Both Surviving Sepsis Campaign and the Centers of Medicare and Medicaid initiated their treatment guidelines to standardize sepsis management. Electronic medical records (EMR) have been widely adopted in health care facilities. EMR that integrated with the Clinical Decision Support system (CDS) for sepsis can play a critical role in improving sepsis management and outcomes.

\section{Definitions of Sepsis}

Since 1992, the definitions of sepsis, severe sepsis, and septic shock have been heavily debated (Kalantari, Mallemat, \& Weingart, 2017). Multiple consensus statements have been released and attempted to incorporate concepts reflecting an updated understanding of the pathophysiology of sepsis (Kalantari et al., 2017). However, none have been perfect or accepted 
as good standard (Kalantari et al., 2017). So far, there have been five definitions of severe sepsis that were published during the past 25 years.

The American College of Chest Physicians (ACCP) and Society for Critical Care Medicine (SCCM) released a consensus statement in 1992 that provided the first published definitions for systemic inflammatory response syndrome (SIRS), sepsis, severe sepsis, septic shock, sepsis-induced hypotension, and multiple organ dysfunction syndrome (MODS) (Figure 1) (Kalantari et al., 2017). Sepsis was diagnosed when at least two metrics or more of the criteria for SIRS. The criteria of SIRS are as following: a) temperature $>38^{\circ} \mathrm{C}$ or $<36{ }^{\circ} \mathrm{C}$, b) heart rate $>90 \mathrm{~min}, \mathrm{c}$ ) respiratory rate $>20 / \mathrm{min}$ or $\mathrm{PaCO}_{2}<32 \mathrm{mmHg}$, and d) white blood cell count $>12,000 / \mathrm{mm}^{3}$ or $<4,000 / \mathrm{mm}^{3}$ or $>10 \%$ immature bands (Bone et al., 1992). Severe sepsis was defined as sepsis complicated by organ dysfunction (Singer et al., 2016). Severe sepsis can progress to septic shock. Septic shock was defined as sepsis-induced hypotension persisting despite adequate fluid resuscitation (Singer et al., 2016). Even though these definitions of sepsis, severe sepsis, and septic shock were not enough to differentiate between life-threatening sepsis and uncomplicated infection, they have been used for more than two decades (Levy et al., 2003).

Figure 1. The definition from ACCP/SCCM Consensus statement, from Kalantari et al. (Kalantari et al., 2017)

\begin{tabular}{|c|c|}
\hline & Definition \\
\hline \multicolumn{2}{|l|}{ SIRS } \\
\hline \multirow[t]{6}{*}{ Criteria } & Two or more of the following \\
\hline & Temperature $>38^{\circ} \mathrm{C}$ or $<36^{\circ} \mathrm{C}$ \\
\hline & Heart rate $>90$ beats per minute \\
\hline & Respiratory rate $>20$ breaths per minute or $\mathrm{PaCO} 2<32 \mathrm{~mm} \mathrm{Hg}$ \\
\hline & White blood cell count $>12,000 / \mathrm{cu} \mathrm{mm},<4,000 / \mathrm{cu} \mathrm{mm}$ or \\
\hline & $>10 \%$ immature (band) forms \\
\hline Sepsis & The systemic response to infection manifested by 2 or more SIRS criteria \\
\hline Severe sepsis & $\begin{array}{l}\text { Sepsis associated with organ dysfunction, hypoperfusion or hypotension that may include but are not limited to, } \\
\text { lactic acidosis, oliguria or an acute alteration in mental status }\end{array}$ \\
\hline Septic shock & $\begin{array}{l}\text { Sepsis-induced with hypotension despite adequate fluid resuscitation along with the presence of perfusion } \\
\text { abnormalities that may include, but are not limited to, lactic acidosis, oliguria, or an acute alteration in mental status. } \\
\text { Patients who are receiving inotropic or vasopressor agents may not be hypotensive at the time that perfusion } \\
\text { abnormalities are measured. }\end{array}$ \\
\hline $\begin{array}{l}\text { Sepsis-induced } \\
\text { hypotension }\end{array}$ & $\begin{array}{l}\text { A systolic blood pressure }<90 \mathrm{~mm} \mathrm{Hg} \text { or a reduction of }>1-40 \mathrm{~mm} \mathrm{Hg} \text { from baseline in the absence of other causes } \\
\text { for hypotension }\end{array}$ \\
\hline MODS & Presence of altered organ function in acutely ill patients such that homeostasis cannot be maintained without intervention \\
\hline
\end{tabular}


In 2001, Dr. Rivers and colleagues proposed the second definition of severe sepsis in their landmark Early Gold Directed Therapy (EGDT) prospective randomized study as follows: a) two of four systemic inflammatory response syndrome criteria, b) systolic blood pressure $\leq 90$ $\mathrm{mmHg}$ (after a crystalloid-fluid challenge of 20 to $30 \mathrm{ml}$ per kilogram of body weight over a 30minute period), and c) blood lactate concentration $\geq$ to $4 \mathrm{mmol} / \mathrm{L}$ (Rivers et al., 2001).

The third definition for severe sepsis was the definition from the International Sepsis Definition Conference led by the Society of Critical Care Medicine (SCCM) from 2001 to 2003 (Faust \& Weingart, 2017). The consensus not only maintained the concepts of sepsis, severe sepsis, and septic shock, as in the report from 1992, but also. provided clinical parameters as a set of criteria for diagnosing sepsis and related conditions (Levy et al., 2003). The consensus concluded that these arbitrary diagnosing criteria would be judged successful if clinicians regard them as an aid for decision-making at the bedside (Figure 2). (Levy et al., 2003).

Figure 2. Diagnosis Criteria for sepsis from 2001 SCCM/ESICM/ACCP/ATS/SIS International Sepsis Definition Conference, from Levy et al. (Levy et al., 2003a)

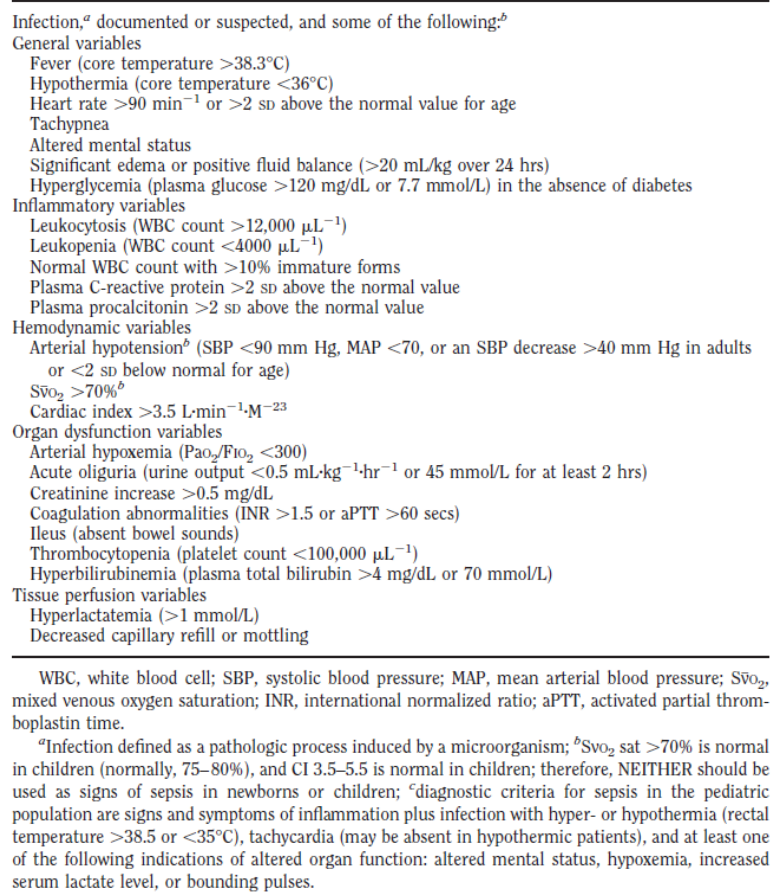


In October 2002, intensive care professionals from around the world initiated the

Barcelona Declaration to reduce the mortality of sepsis with the initiation of the Surviving Sepsis

Campaign (SCC). The SSC was leading by the European Society of Intensive Care Medicine

(ESICM), SCCM, and the International Sepsis Forum. The SCC provided the fourth definition of severe sepsis and initiated the sepsis bundles guidelines for sepsis management as a world-wide standardized protocol (Figures 3 and 4)(Dellinger et al., 2013). In 2004, the first guideline for management of severe sepsis and septic shock was published, with revisions in 2008, 2012,

2016, and 2018. The SCC guideline was a source for the qualifiers of end-organ damage that was later adopted by NQF \#0500 and the SEP-1 measure (Faust \& Weingart, 2017).

Figure 3 Definition of sepsis, from Dellinger et al. (Dellinger et al., 2013)

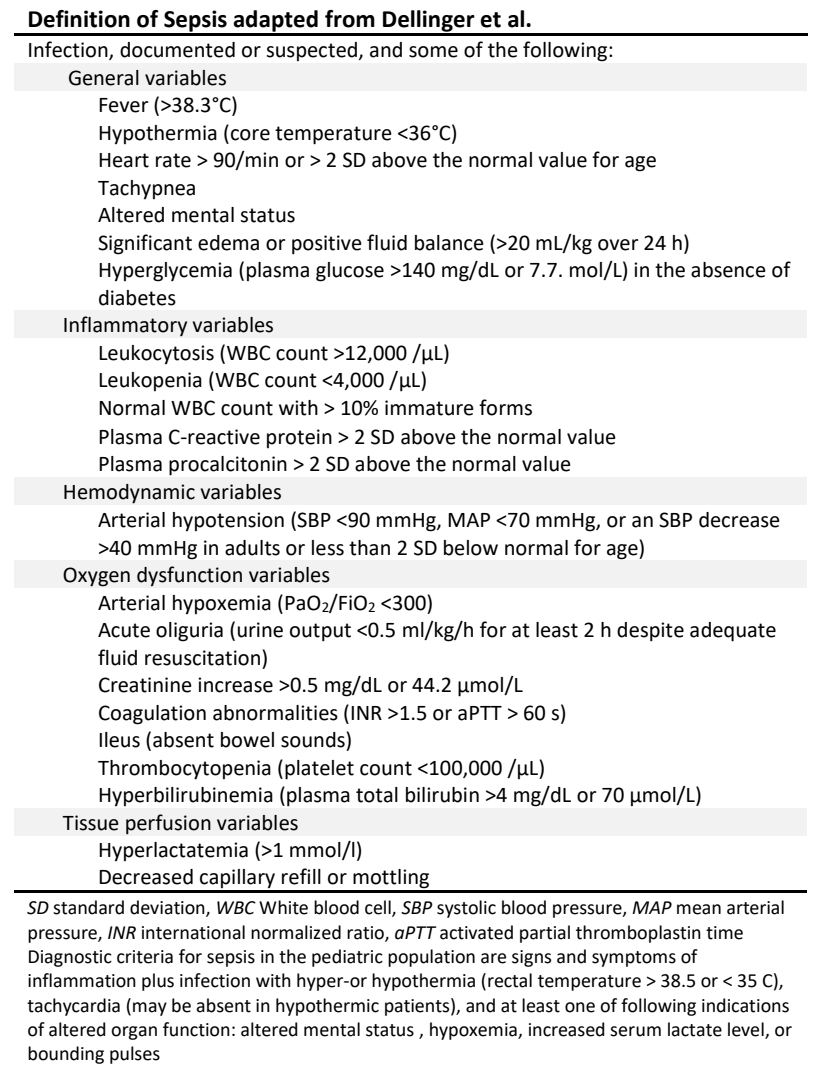


Figure 4 Definition of severe sepsis, from Dellinger et al. (Dellinger et al., 2013)

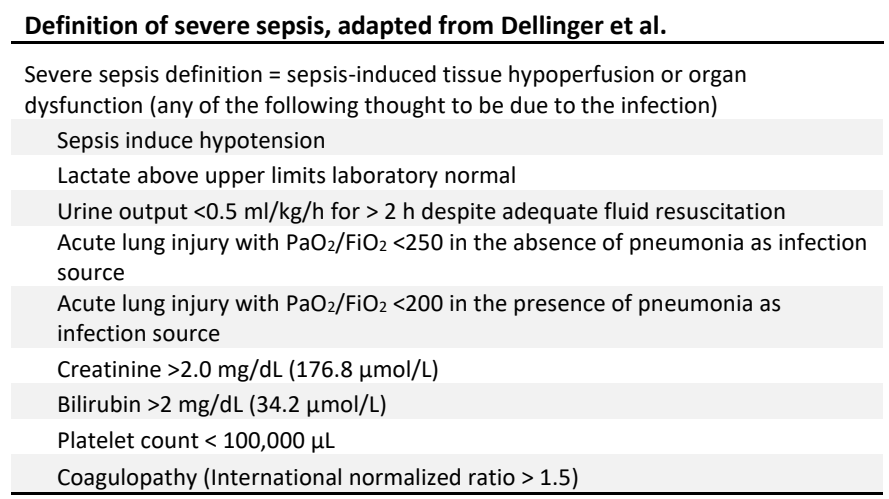

In 2014, ESICM and SCCM convened a task force of 19 critical care, infectious disease, surgical, and pulmonary specialists to reexamine the current and up-to-date definition of sepsis. The task force recognized that sepsis was a syndrome without, at present, a validated criterion standard diagnosis test (Singer et al., 2016). In 2016, the task force released the fifth consensus definition of sepsis, Sepsis-3, defining sepsis as life-threatening organ dysfunction caused by a dysregulated host response to infection (Singer et al., 2016). The new definition emphasizes the primary host response to infection, the potential lethality, and the need for urgent recognition (Singer et al., 2016).

The deterioration of sepsis eventually leads to organ dysfunction and failure. Many scoring systems have been initiated to quantify the severity of organ dysfunction by using clinical findings, laboratory data, or therapeutic interventions. One of the most recognizable criteria to quantify the dysfunctionality of organs is the Sequential Organ Failure Assessment (SOFA) that has been used widely in critical care settings but not in other specialties (Figure 5). The task force recommended using change in the baseline of the total SOFA score of 2 points or more to represent organ dysfunction (Singer et al., 2016). 
Figure 5. Sequential (Sepsis-Related) Organ Failure Assessment Score (SOFA), adapted from Singer et al. and Vincent et al. (Singer et al., 2016; Vincent et al., 1996)

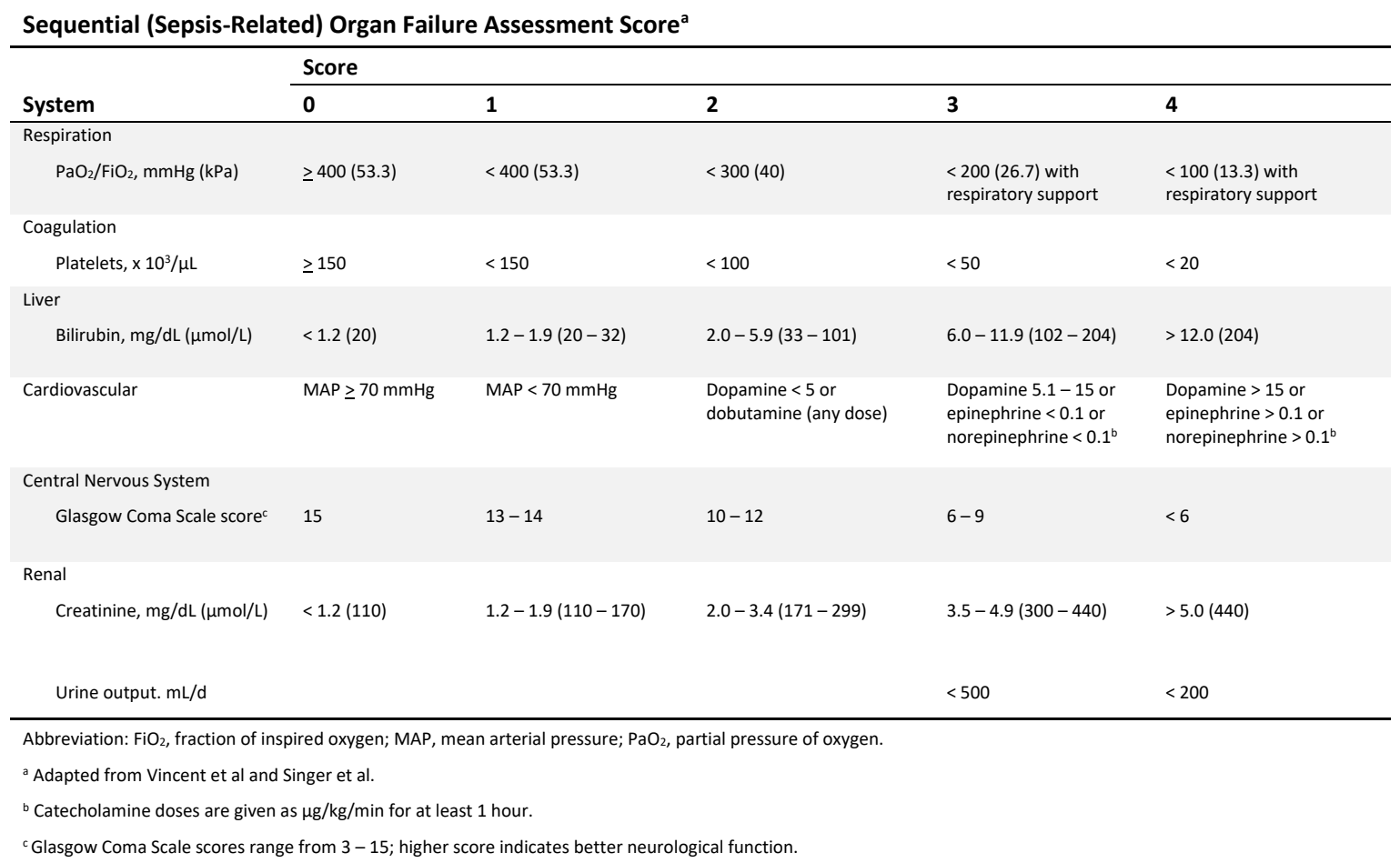

The task force eliminated the severe sepsis category and defined septic shock as a subset of sepsis in which underlying circulatory ad cellular metabolism abnormalities were profound enough to substantially increase mortality (Singer et al., 2016). The septic shock was identified when a patient with sepsis developed persisting hypotension requiring vasopressor to maintain MAP > $65 \mathrm{mmHg}$, and having serum lactate level > $2 \mathrm{mmol} / \mathrm{L}(18 \mathrm{mg} / \mathrm{dl})$ despite adequate volume resuscitation (Singer et al., 2016).

The SOFA score required laboratory testing and thus may not promptly capture dysfunction in individual organ system (Singer et al., 2016). The task force recommended the quick Sequential Organ Failure Assessment (qSOFA) criteria that do not require laboratory tests and can be assessed quickly and repeatedly (Singer et al., 2016). The change in the baseline of the total SOFA score of 2 points or more to represent organ dysfunction (Singer et al., 2016). 
The qSOFA score can promptly detect deteriorating patients and lead a physician to further investigate and monitor for organ dysfunction, to initiate or escalate therapy as appropriate, and to consider referral to critical care or increase the frequency of monitoring, if such actions have not already been undertaken (Singer et al., 2016). The qSOFA criteria are a) respiratory rate $\geq$ 22/min, b) altered mental status, and c) systolic blood pressure $\leq 100 \mathrm{mmHg}$ (Figure 6) (Singer et al., 2016).

Figure 6. Quick Sequential Organ Failure Assessment (qSOFA)

\section{Quick Sequential Organ Failure Assessment (qSOFA)}

Altered mental status

Systolic blood pressure $\leq 100 \mathrm{mmHg}$

Respiratory rate $\geq 22$ breaths per minute

Sepsis is a life-threatening condition that merits prompt detection and intervention. The failure to meet two or more qSOFA or SOFA criteria should not lead to a deferral of investigation or treatment of infection or to a delay in any aspect of care deemed necessary by the practitioners (Singer et al., 2016). The development of the definition and criteria of sepsis and sepsis-related conditions has been an integral part of the development of tools to detect, categorize, screen, alert, capture, and facilitate the management of sepsis. The early detection of sepsis will lead to early intervention for this fatal condition.

\section{Early Goal-Directed Treatment of Severe Sepsis/Septic Shock}

In the early 1990, the Early Goal-Directed Therapy (EGDT) Collaborative Group challenged the paradigm of sepsis care as an "ICU disease" by applying similar urgent diagnosis and therapeutic principles as used for myocardial infarction, stroke, and trauma at the point of presentation in the emergency department (H. B. Nguyen et al., 2016). EGDT comprised of early identification of high-risk patients, appropriate cultures, source control, administration of 
antibiotics, followed by initial hemodynamic optimization of oxygen delivery (H. B. Nguyen et al., 2016). In 2001, Dr. Rivers, who pioneered the EGDT at Henry Ford Hospital, reported a significant reduction in mortality $(30.5 \%$ vs. $46.5 \%)$ among patients with severe sepsis and septic shock who were treated according to a 6-hour protocol of EGDT when compared with standard therapy (Rusconi et al., 2015). The clinical benefit of EGDT prompted the SSC to develop sepsis care bundles based on the initial resuscitation of EGDT, and published in 2004 (Figure 7)

(Allison \& Schenkel, 2018). The SSC sepsis management resuscitation bundle was adopted internationally as the standard of care for early sepsis management (H. B. Nguyen et al., 2016). In 2008, the National Quality Forum (NQF) proposed sepsis bundle NQF \#0500 based on the EGDT.

Figure 7. Surviving Sepsis Care Bundles 2004 (Dellinger et al., 2004)

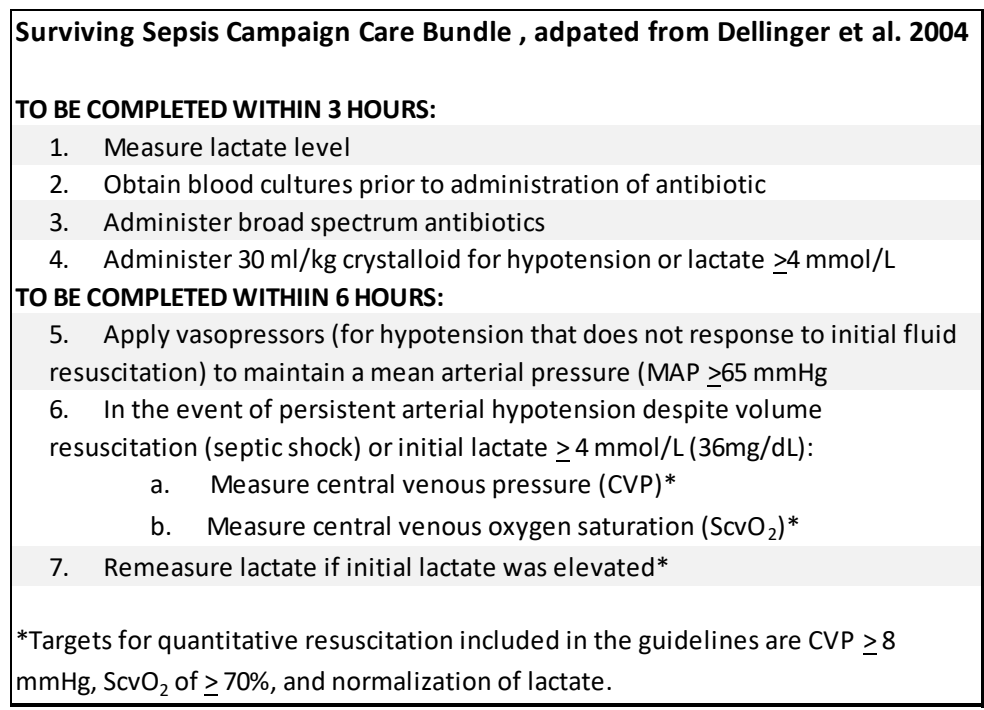

The initiatives that focused on early recognition and treatment included legislation passed in 2014 in New York requiring hospitals to report a variety of sepsis process measures to the New York State Department of Health (NYSDOH), and the CMS (Novosad et al., 2016). NYSDOH required all hospitals to submit and follow evidence-informed protocols (including 
elements of 3-hour and 6-hour sepsis bundles: lactate measurement, early blood cultures and antibiotic administration, fluids, and vasopressor) for early identification and treatment of severe sepsis and septic shock (Levy, Gesten, et al., 2018). Levy and colleagues reported the results of the first two years of the program that the compliance with elements of the 3-hour and 6-hour sepsis bundles increased significantly over time while the risk-adjusted mortality significantly decreased from $28.8 \%$ to $24.4 \%$ (p < 0.001) (Levy, Gesten, et al., 2018). Levy concluded that the risk-adjusted sepsis mortality decreased during the initiative was associated with increased hospital-level compliance (Levy, Gesten, et al., 2018). Furthermore, NYDOH data also showed that the increased risk-adjusted in-hospital mortality was associated with a longer time to complete the 3-hour bundle and longer time to administration of antibiotics (Seymour et al., 2017).

Several randomized control trials did not confirm the survival benefit of EDGT when compared with the standard of care. Between 2014 and 2015, three landmark separate randomized controlled trials were published; a) Protocol-Based Care for Early Septic Shock (ProCESS) (Yealy et al., 2014), b) Protocolized Management in Sepsis (ProMISe) (Mouncey et al., 2015), and c) Australian Resuscitation in Sepsis Evaluation (ARISE) (Rowan et al., 2017). All three trials reported that there was no statistical difference in morbidity and mortality between EGDT bundles and the standard of care for patients with sepsis (Esposito et al., 2018). Systematic review and meta-analysis reported concurred with the results of the three landmark trials that in-hospital mortality did not differ between EGDT and usual care (Rusconi et al., 2015).

Surviving Sepsis Campaign has developed guidelines for the definition of sepsis and treatment since 2004. The guidelines were revised in 2008, 2012, 2016, and 2018 based on 
updated new development of definitions, literature, pathophysiology, and new treatment technology. The first SSC guideline that published in 2004 based on the aggressive initial resuscitation of EGDT that had recommendations for interventions to be completed in the first 3and 6 hours. Every aspect of the guidelines has been debated, studied, challenged, and revised to find the best of the standard practices. The subsequent revision of the Surviving Sepsis guidelines moved from a protocolized quantitative resuscitation strategy to a more patientcentered resuscitation approach guided by hemodynamic assessment and ongoing reevaluation of the response to treatment (De Backer \& Dorman, 2017). The mainstays of the treatment remained to be infection source control and early antibiotic therapy. The latest SSC guideline that was published in 2018 emphasized that sepsis was a medical emergency that necessitates an urgent assessment and treatment. The most significant change in the revision of the SSC bundles was the 3-hour, and 6-hour bundles had been combined into a single "hour-1 bundle" with the explicit intention of beginning resuscitation and management immediately (Figure 8) (Levy, Evans, \& Rhodes, 2018). SSC concluded that the update guidelines should be introduced to the emergency department, floor, and ICU staff as the next iteration of ever-improving tools in the care of patients with sepsis and septic shock as we all work to lessen the global burden of sepsis (Levy, Evans, et al., 2018).

Figure 8 Hour-1 SSC (Levy, Evans, et al., 2018)

\section{Hour-1 Surviving Sepsis Campaign Bundle of Care*}

. $\quad$ Measure lactate level. Remeasure if initial lactate is $>2 \mathrm{mmol} / \mathrm{L}$
. $\quad$ Administer broad-spectrum antibiotics
Apply vasopressors if patient is hypotensive during or after fluid resuscitation to maintain MAP $\geq 65 \mathrm{mmHg}$
*Adapted from Levy et al.
**“Time zero" or "time of presentation" is defined as the time of triage in Emergency Department or if presenting
from another care venue, from the earliest chart annotation consistent with all elements of sepsis (formerly severe
sepsis) or septic shock ascertained through chart review.




\section{Early Management Bundle for Severe Sepsis/Septic Shock (SEP-1)}

In October 2015, the Centers for Medicare and Medicaid Services (CMS) enacted a National Quality Measure (NQF \#0500) for reporting on sepsis called the Early Management Bundle for Severe Sepsis/Septic Shock (SEP-1) (Figure 9) (Faust \& Weingart, 2017). SEP-1 was created to standardize sepsis care in the United States to improve the outcomes of sepsis (Allison \& Schenkel, 2018). Sep-1 is the nation's first, and by law only, national quality measure on early management of sepsis (Faust \& Weingart, 2017). Sep-1 mandates that all patients at an emergency department whom meeting criteria for SEP-1 must receive the bundle of care stipulated in the CMS Specifications Manual for National Hospital Inpatient Quality Measures (Faust \& Weingart, 2017). Medicare included the SEP-1 Quality Measure in the Medicare Hospital Compare Reports, which is a publicly available database rating on a hospital, based on each CMS captured measure (Faust \& Weingart, 2017). The metric of interest to CMS for SEP-1 was adherence to the measure, not mortality or other patient-centered outcome (Faust \& Weingart, 2017). Because it was a priori assumption that adherence to the quality measure improved mortality (Faust \& Weingart, 2017). Many data elements of the SEP-1 were updated, made optional on the following updated version in 2018. 
Figure 9. The criteria of SEP-1, adapted from Faust \& Weingart (Faust \& Weingart, 2017).

Data and Time Elements of the SEP-1 Measure

\begin{tabular}{|c|c|c|}
\hline \multirow[b]{2}{*}{ Time } & \multicolumn{2}{|c|}{ Numerator } \\
\hline & Severe sepsis & Septic Shock \\
\hline 3-h bundle elements & $\begin{array}{l}\text { 1. Initial lactate level measurement } \\
\text { 2. Broad-spectrum or other antibiotic } \\
\text { administered } \\
\text { 3. Blood cultures drawn prior to antibiotics and } \\
\text { received within } 6 \text { h of severe sepsis presentation }\end{array}$ & $\begin{array}{l}\text { 1. All severe sepsis } 3-\mathrm{h} \text { bundle elements } \\
\text { and } \\
\text { 2. Resuscitation with } 30 \mathrm{~mL} / \mathrm{kg} \text { crystalloid fluids }\end{array}$ \\
\hline 6-h bundle elements & $\begin{array}{l}\text { 1. Repeat lactate level measurement only if initial } \\
\text { lactate level is elevated }\end{array}$ & $\begin{array}{l}\text { 1. Vasopressor agents only if hypotension persists after } \\
\text { fluid administration } \\
\text { 2. Only if hypotension persists after fluid administration } \\
\text { or initial lactate level } \geq 4 \mathrm{mmol} / \mathrm{L} \text { (within } 6 \mathrm{~h} \text { or } \\
\text { presentation of septic shock), then repeat volume status } \\
\text { and tissue perfusion assessment consisting of either: } \\
\text { a. Focused examination including: vital signs and } \\
\text { cardiopulmonary examination and capillary refill } \\
\text { evaluation and peripheral pulse evaluation and skin } \\
\text { examination } \\
\text { b. Any two of the following four measurements: } \\
\text {. Central venous pressure measurement } \\
\text {. Central venous oxygen measurement } \\
\text {. Bedside cardiovascular ultrasonography } \\
\text {. Passive leg raise or fluid challenge }\end{array}$ \\
\hline \multicolumn{3}{|l|}{ Denominator } \\
\hline \multicolumn{3}{|l|}{ Included: } \\
\hline $\begin{array}{l}\text { 1. Age } \geq 18 y \\
\text { 2. Inpatient } \\
\text { 3. ICD-10-CM princip }\end{array}$ & al or other diagnostic code of sepsis, severe sepsis, & or septic shock \\
\hline \multicolumn{2}{|l|}{ Excluded: } & 1. Directive for comfort care or palliative care within $3 \mathrm{~h}$ of presentation of severe sepsis \\
\hline \multicolumn{3}{|c|}{ 2. Directive for comfort care or palliative care within $6 \mathrm{~h}$ of presentation of septic shock } \\
\hline \multicolumn{3}{|c|}{ 3. Administrative contraindication to care within $6 \mathrm{~h}$ of presentation of severe sepsis } \\
\hline \multicolumn{3}{|c|}{ 4. Administrative contraindication to care within $6 \mathrm{~h}$ of presentation of septic shock } \\
\hline \multicolumn{3}{|c|}{ 5. Length of stay $>120 \mathrm{~d}$} \\
\hline \multicolumn{3}{|c|}{ 6. Transfer from another acute-care facility } \\
\hline \multicolumn{3}{|c|}{ 7. Patients with severe sepsis who die within $3 \mathrm{~h}$ of presentation } \\
\hline \multicolumn{3}{|c|}{ 8. Patients with septic shock who die within $6 \mathrm{~h}$ of presentation } \\
\hline \multicolumn{3}{|c|}{ 9. Patients receiving IV antibiotics for more than $24 \mathrm{~h}$ prior to presentation of severe sepsis } \\
\hline
\end{tabular}

ICD-10-CM = International Classification of Diseases, Tenth Revision, Clinical Modification. 
The SEP-1 has created a lot of debates since the launch in 2015. The first controversy of SEP-1 was the definition of sepsis. CMS's definition of sepsis derived from the SCC and NQF, but CMS definitions independently lowered the threshold of widely accepted studies for lactate levels (Kalantari et al., 2017). According to CMS, lactate $>2 \mathrm{mmol} / \mathrm{L}$ represented a patient with severe sepsis, and initial lactate $>4 \mathrm{mmol} / \mathrm{L}$ defined a patient with septic shock, in which the change came without supporting evidence (Kalantari et al., 2017). A primary concern regarding the definition of severe sepsis in SEP-1 was the potential for excessive use of broad-spectrum antibiotics, which was the crux of complaint from the Infectious Diseases Society of America (IDSA) (Faust \& Weingart, 2017). IDSA believed that the SEP-1 represented a significant threat to antibiotic stewardship by not only it would lead to antibiotics shortage and increased drugresistant organisms, but also exacerbated C. Difficile infection (Faust \& Weingart, 2017). Furthermore, Esposito reported that the SEP-1 measure associated with a significant increase in the utilization of resources as well as the cost for both patients and hospitals without improvement in mortality (Esposito et al., 2018).

The second controversy of SEP-1 was the hemodynamic intervention protocol. SEP-1 did not reflect the best evidence in the management of early severe sepsis and septic shock (Faust \& Weingart, 2017). The lack of survival benefits when using the SEP-1 measure also reported in several studies (Esposito et al., 2018; Pepper, Natanson, \& Eichacker, 2018). A systematic review by Pepper et al. evaluated hemodynamic intervention of SEP-1 and showed only lowlevel evidence supporting survival benefit with serial lactate measurement or a $30 \mathrm{~mL} / \mathrm{kg}$ fluid infusion (Pepper et al., 2018). The concern about fluid resuscitation was that it should be cautiously introduced to each patient with careful assessment to prevent fluid overload which can be fatal. 
The third controversy of the SEP-1 measure was the complexity and burdensome of reporting. The initial SEP-1 measure was a 51-page specification manual accompanied by a 393page guide, which required documentation of adherence to 141 specific actions or variables represented by 20 separate flowcharts with multiple decision points (Faust \& Weingart, 2017). The SEP-1 was one of CMS's most complex performance measures that required documentation of tasks for one patient and could take as long as three hours (Pepper et al., 2018).

Given the lack of convincing evidence that patients benefited from adherence to the SEP1 bundle, low compliance among respondent hospitals should not be surprising (Allison \& Schenkel, 2018). Venkatesh and colleagues reported that only $54 \%$ of patients presenting with severe sepsis or septic shock were treated in full compliance with the SEP-1 bundle in hospitals with a self-reported interest in sepsis care (Venkatesh et al., 2018). Barbash et al. (2017) reported that among hospitals reporting SEP-1 performance data, overall bundle compliance was low, but it varied widely across hospitals (mean and SD $48.9 \% \pm 19.4 \%$ ) (Barbash, Rak, Kuza, \& Kahn, 2017).

The study by Rhee et al. showed that the crude mortality rates were higher in sepsis cases that failed SEP-1 when comparing that with passed SEP-1, but there was no difference after adjusting for clinical characteristics and severity of illness (Rhee et al., 2018). Rhee's study also showed that delays of greater than 3 hours until antibiotics were significantly associated with death but only accounted for a small fraction of SEP-1 failures (Rhee et al., 2018).

Rapid identification of sepsis, early intervention of hemodynamic support with fluids, prompt administration of appropriate antimicrobial therapy and monitoring of clinical and hemodynamic parameters are crucial elements to be considered in the treatment of patients with severe sepsis or septic shock, especially in patients with a high baseline risk of mortality 
(Rusconi et al., 2015). Until further evidence exists, it is still reasonable to consider EGDT (Rusconi et al., 2015). So far, there were several controversies about the SEP-1 and the compliance was low in general. If we could increase compliance with the measure, we might see an improvement in the outcomes of treatment. 


\section{Chapter 2 Literature review}

\section{Clinical decision support and sepsis}

Clinical Decision Support (CDS) is all about intelligence: clinical knowledge and data intelligently applied at the point where healthcare decisions are made (Osheroff, J.A., Teich, J.M., Levick, D., Saldana, L., Velasco, F.T., Sittig, D.F., .. \& Jenders, R.A., 2012). CDS has been defined as a process for enhancing health-related decisions and actions with pertinent, organized clinical knowledge and patient information to improve healthcare and healthcare delivery (Campbell, 2013). CDS is an essential tool to review and assess the data from a variety of sources, trigger the notification to raise awareness, and guide intervention. The framework for configuring and deploying effective CDS implementation is the Five Rights approach; a) the right information, b) to the right person, c) in the right intervention format, d) through the right channel, and e) at the right time workflow (Campbell, 2013). An ideal sepsis CDS is available in real-time and at the point of care, integrating cloud-based CDS with the host electronic health record (EHR) system in a patient-centric clinical workflow (Dixon et al., 2013). There are four categories of CDS interventions; a) data entry, b) data review, c) assessment and understanding, and d) triggered by user task (Osheroff, J.A., Teich, J.M., Levick, D., Saldana, L., Velasco, F.T., Sittig, D.F., ... \& Jenders, R.A., 2012). The examples of the data entry category are smart forms and order sets. Order sets are a key tool in the CDS arsenal because they are thought to reduce medical errors, and enhance workflow (Campbell, R. J., 2016). The example of data review category is the Virtual ICU for patient monitoring. The example of the assessment and understanding category is the Health Level Seven (HL7) Infobutton that links to detailed, evidence-based knowledge regarding the disease and its treatment (Campbell R. J., 2016). The 
example of triggered by user task category is an alert in EMR, text message, or electronic mail notification (Campbell, R. J., 2016).

Sepsis is an enigmatic clinical syndrome that arises when a patient reacts adversely to infection and develops organ dysfunction as a consequence (Handbook of Sepsis, 2018). Initial clinical presentation of sepsis can be non-specific and obscured by underlying morbidity of a patient, which makes sepsis challenging to detect until the condition becomes deteriorated. The sepsis criteria alone can indicate non-specific organ dysfunction and must be used in combination with clinical judgment. Given the difficulties in the timely recognition of sepsis, healthcare institutions have been increasingly leveraging clinical data captured in EMR and CDS systems that incorporated patient-specific context such as vital signs and laboratory values to alert clinicians to the possible presence of sepsis and other clinical deteriorations (Downing et al., 2019).

Many retrospective studies focused on development of CDS tool for sepsis screening and alert (Amland \& Hahn-Cover, 2016; Amland, Haley, \& Lyons, 2016; Brown et al., 2016; Li et al., 2019; S. Q. Nguyen et al., 2014; Rolnick et al., 2016). Automated screening tools have the potential to decrease diagnostic delays and increase screening accuracy (Bhattacharjee, Edelson, \& Churpek, 2017). The most frequent acute care e-alerts were designed for early identification of systemic inflammatory response syndrome, sepsis, severe sepsis, or septic shock as a combined group (Benthin, Pannu, Khan, \& Gong, 2016). Clinical trials on the effectiveness of e-alerts have been shown mixed results, with high sensitivity and poor specificity, and this may be a reason for decreases efficacy (Benthin et al., 2016). CDS alerts with high sensitivity, but low specificity can cause alert fatigue after too many notifications, and the alert can be overridden by the providers. The accuracy of the automated sepsis screening varied from sensitivity $17.1 \%$ to $98.9 \%$, 
specificity $18.1 \%$ to $96.7 \%$ (Makam, Nguyen, \& Auerbach, 2015). The systematic review reported that automated electronic sepsis alert systems had poor positive predictive value (20.5\%-53.8\%) and did not improve mortality nor length of stay (Makam et al., 2015).

Several studies tried to improve the accuracy of the sepsis screening tool by incorporating additional clinical data and using an application of machine learning and artificial intelligence (AI) in data analytics. Several studies evaluated the predictability of machine learning-based CDS tool for sepsis detection using SIRS criteria, as well as additional clinical and laboratory information (Gultepe et al., 2014; Horng et al., 2017; Shimabukuro, Barton, Feldman, Mataraso, \& Das, 2017; Taylor et al., 2016). Horng and colleagues reported that adding free text from clinical notes in the model development of the CDS tool improved discriminating ability (Horng et al., 2017). The randomized trial by Shimabukuro and colleagues reported significant benefits of the length of stay and mortality with the use of machine learning-based CDS tools to predict severe sepsis (Shimabukuro et al., 2017). Nemati et al. reported that the machine learning model (AI) Sepsis Expert for real-time sepsis prediction in ICU could accurately predict the onset of sepsis 4-12 hours before clinical recognition (Nemati et al., 2018). Giannini and colleagues reported that the machine learning algorithm could predict the impending occurrence of severe sepsis and septic shock with low sensitivity but high specificity (Giannini et al., 2019).

The sepsis screening is only a part of a complex clinical pathway of sepsis management that requires the coordination of multidisciplinary teams. Some studies evaluated not only the accuracy of sepsis screening tools but also the process of sepsis care by incorporating recommended standard sepsis management. Manaktala and colleagues studied before and after implementation of sepsis improvement program in inpatient units, which consisted of a combination of a) sepsis education, b) process improvement through change management, and c) 
an electronic CDS system (Manaktala \& Claypool, 2017). The electronic CDS system conducted real-time surveillance of EMR data and delivered both alerts to the nursing staff's mobile devices at the point of care and SSC sepsis management recommendation (Manaktala \& Claypool, 2017). Manaktala's study concluded that the sepsis improvement program significantly decreased mortality (Manaktala \& Claypool, 2017).

A randomized trial by Semler evaluated the benefit of electronic sepsis assessment and management tools in ICU (Semler et al., 2015). The integrated sepsis assessment and management tool contained laboratory results and normal range, vital signs, assessment tab with criteria of severity of sepsis, and management tab with recommended SSC guidelines and "single click" order entry (Semler et al., 2015). The patients were randomized between the sepsis assessment and management tool and usual care. Semler's study did not find significant differences in time to completion of SSC 6-hr resuscitation bundle elements, time to complete each element individually, ICU mortality, ICU free days, ventilator-free days, or vasopressorfree days. Furthermore, the study reported that the utilization of the tool was low $(28.4 \%)$ (Semler et al., 2015).

Clinical decision support (CDS) can significantly impact improvements in quality, safety, efficiency, and effectiveness of health care (HealthIT.org, n.d.). Many organizations are facing significant challenges when it comes to creating a CDS tool that is intuitive, user-friendly, and effective protocols for alarms, alerts, and decision-making pathways (Bresnick, J. 2017). Alert fatigue and clinical burnout are common byproducts of poorly implemented CDS features that overwhelm users with unimportant information or frustrating workflow freezes that require an extra click to circumvent (Bresnick, J. 2017).

In 2011, Ochsner Health System launched electronic medical records at all its medical 
centers and health clinics. Since then Ochsner has been actively engaged the CDS applications in many clinical settings. Order sets for many clinical settings were created and integrated in the EMR. We believed that the order set would streamline the treatment process, reduce error, and helpe the providers to adhere to the treatment protocols. Ochsner Medical Center has reported the CMS SEP-1 measure since the launch in 2015. The treatment guideline for CMS measure has been incorporated in the EMR in the form of the sepsis order set. The sepsis order set contains a package of antibiotic recommendations, laboratory orders, and other recommended treatment guidelines based on standard of care by SEP-1 measure. Providers can find the sepsis order set in the list of order sets in the EMR. However, the medical center experienced low usage of the sepsis order set as well as low compliance of SEP-1 measure which below Louisiana average and National average. Ochsner aimed to improve the quality of patient care, increase performance metrics, and standardize the treatment by promoting adherence to CMS SEP-1. In January 2018, Ochsner's informatic team launched the sepsis screening tool to raise awareness of the providers and encourage the use of sepsis order set. The purposes of this study were to determine whether the sepsis screening tool increases the usage of sepsis order set. We believed that the usage of sepsis order set would increase the compliance of the SEP-1 measure and eventually, improve the treatment outcomes. The primary outcomes were a) time zero to antibiotics, b) inpatient length of stay, and c) survival at discharge. 


\section{Chapter 3 Materials and Methods}

\section{Study design and population}

This retrospective cohort study conducted at the main facility of a tertiary academic hospital (Ochsner Medical Center, New Orleans, LA). Data were extracted electronically from Ochsner's EMR. The population was adult patients who admitted through the emergency department and had a diagnosis of sepsis or septic shock during admission from July $1^{\text {st }}, 2017$ to July $31^{\text {st }}, 2018$. The diagnoses of sepsis/septic shock based on the International Classification of Disease (ICD)-10. The time that a patient arrived at the emergency department was the time zero. The exclusion criteria were a) patients who were referred from outside facilities, b) who had antibiotics started before arrival at the emergency department, and c) who were transferred to outside facilities.

At the emergency department, a triage nurse will evaluate every patient and then enter a patient's clinical data in the EMR. If a patient were considered to have sepsis, he or she would be brought in to have prompt evaluation and intervention by the emergency care team. In January 2018, the sepsis screening tool was launched and incorporated into EMR at the nurse triage station. The sepsis screening tool contained clinical history and screening criteria based on SIRS criteria (Figure 10). After the launch of sepsis screening tool, a triage nurse would go through an additional step of checking the sepsis criteria list. If there were positive at least 2 out of 3 criteria, a patient would be considered to have sepsis. The sepsis screening tool would trigger automated alerts banner in the provider's EMR about arriving of the septic patient. The EMR equipped with the list of order sets for different clinical settings, including the sepsis order set. The sepsis order set contains a package of treatments included antibiotics recommendation, laboratory testing orders, and other recommended standard management based on SEP-1 measure. The sepsis order 
set does not have a direct link from the sepsis screening tool. The use of order set was optional.

This study collected patients' data during 6 months before and 6 months after the launch of the sepsis screening tool, which would be called the preintervention and postintervention group respectively. The compliance with each element of SEP-1 measure at 3-hour and 6-hour was collected based on the eligibility of patient's conditions that met the criteria for the intervention. The perfect care was achieved when a patient received all required elements according to the protocol at 3 -hour (3H perfect care) and 6-hour $(6 \mathrm{H}$ perfect care). The total perfect care was the group of patients who completed the required bundle elements at the indicated time frame at both 3- and 6- hour. Total perfect care represented the compliance to SEP-1 measure and was used for reporting to CMS Hospital Compare. The primary outcomes were a) the duration from the arrival at the emergency department to the time that the antibiotic was given to a patient (time zero to antibiotic), b) inpatient length of stay, and c) survival rate at discharge.

Figure 10. The sepsis screening tool

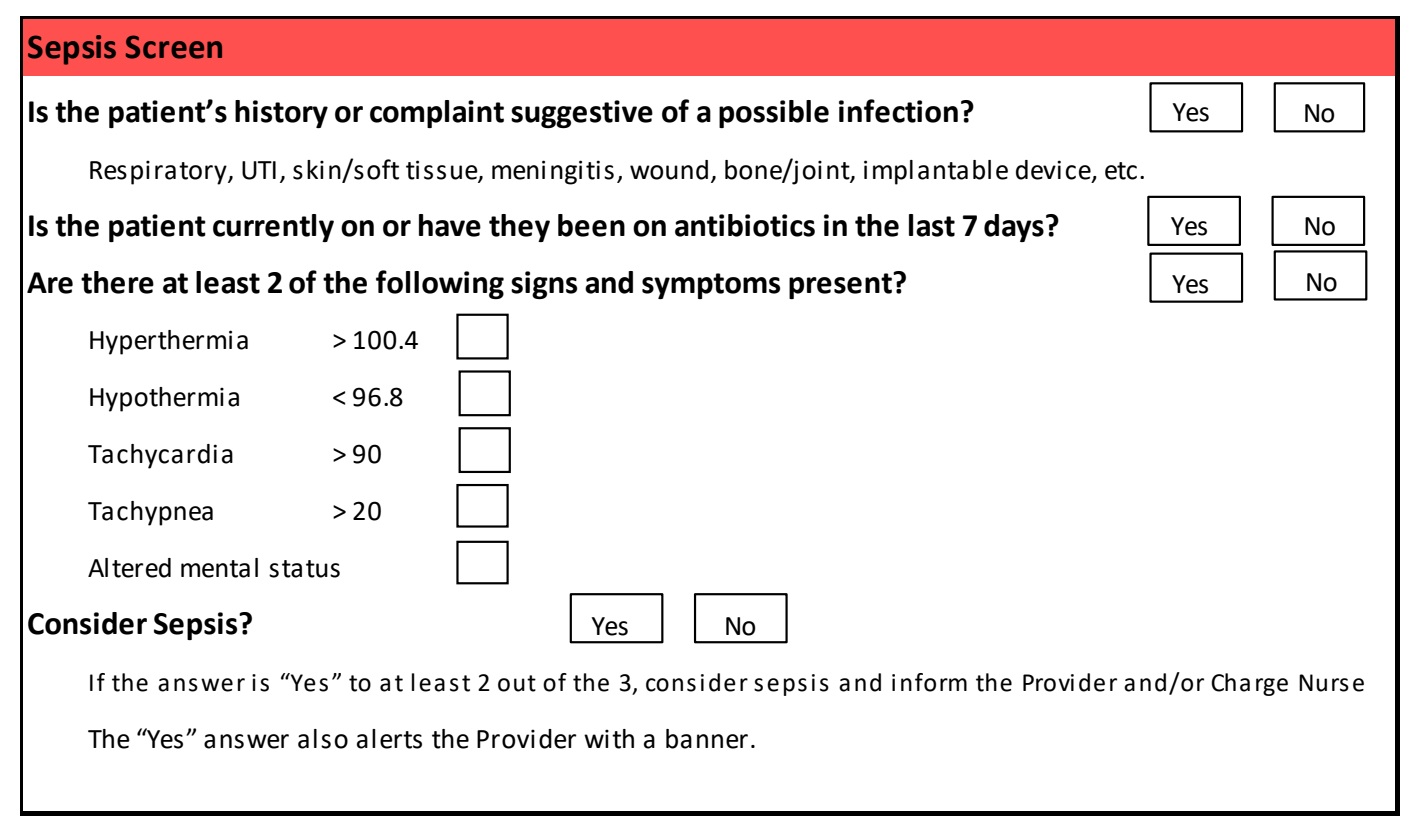


We compared preintervention and postintervention group to determine whether the sepsis screening tool increase the usage of sepsis order set, increase compliance of the SEP-1 measure, and to assess the association between the launch of sepsis screening tool and the primary outcomes. We believed that when the order set was used, the treatment guidelines would be followed correctly and completely. We regrouped the whole population of 632 to be the group that used sepsis order set and did not use the sepsis order set. Then we assess the association between using sepsis order set and total perfect care, and the association between sepsis order set and the primary outcomes. We believed that the adherence to the quality measure improved outcomes of the treatment. We regrouped the whole population of 632 into the group that achieved total perfect care and did not achieve total perfect. Then we assessed the association between total perfect care and the primary outcomes.

This study was approved by the University of Tennessee Institutional Review Board as meeting criteria for exempt status for nonhuman subjects research status; 18-05810-NHRS.

\section{Statistical analysis}

We used the Chi-square statistic to assess the association between the categorical variables for the launch of the sepsis screening tool and the order set use, the launch of the sepsis screening tool, and total perfect care, and the order set usage and total perfect care. Independent $t$-test was used to assess the association between time zero to antibiotic and a) the launch of sepsis screening tool, b) order set use, and c) total perfect care. We used the negative binomial regression statistic to assess the association between the inpatient length of stay and a) the launch of sepsis screening tool, b) order set use, and c) total perfect care. Logistic regression statistic was used to assess the association between the survival at discharge and a) the launch of sepsis screening tool, b) order set use, and c) total perfect care. All analyses were performed using 
SPSS version 26 (IBM Corp., Armonk, NY). The $p$-value of less than 0.05 was considered to be statistically significant. 


\section{Chapter 4 Research Results}

The final population for analysis was 632 , in which there were 260 patients during the six months before the launch of the sepsis screening and 372 patients during the six months after the launch. The study cohort is shown in figure 11 . The collected data before and after the launch of the sepsis screening tool is shown in figure 12.

Figure 11. The study cohort.

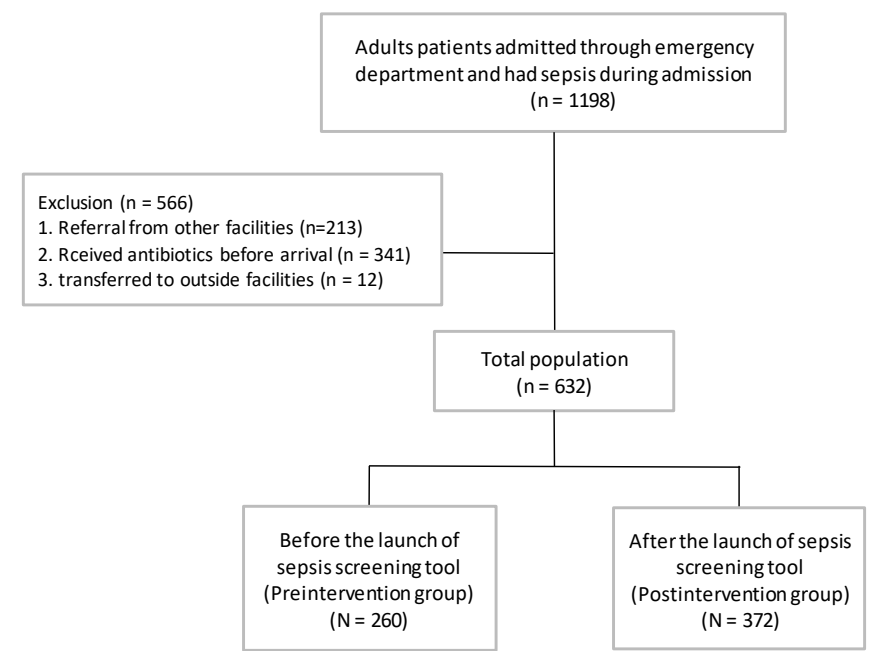

Figure 12. The table comparing before and after the launch of the sepsis screening tool

\begin{tabular}{|c|c|c|c|c|c|}
\hline \multirow[b]{2}{*}{ Order set use } & \multicolumn{2}{|c|}{ Preintervention group } & \multicolumn{2}{|c|}{ Postintervention group } & \multirow[b]{2}{*}{$p=0.001$} \\
\hline & $135 / 260$ & $51.72 \%$ & $242 / 372$ & $65.05 \%$ & \\
\hline Time zero to antibiotic: average & $135.15 \mathrm{~min}$ & $(1-1262)$ & $117.5 \mathrm{~min}$ & $(2-1580)$ & \\
\hline Inpatient length of stay: average & 6.25 days & $(0.28-55.75)$ & 6.23 days & $(0.29-55.35)$ & \\
\hline Survival rate at discharge & $234 / 260$ & $90 \%$ & $347 / 372$ & $93.28 \%$ & \\
\hline 3H perfect care & $155 / 260$ & $59.39 \%$ & $257 / 372$ & $69.09 \%$ & \\
\hline $3 \mathrm{H}$ lactate & $240 / 260$ & $91.95 \%$ & $350 / 372$ & $94.09 \%$ & \\
\hline Blood Culture & $229 / 260$ & $87.74 \%$ & $338 / 372$ & $90.86 \%$ & \\
\hline Antibiotics & $208 / 260$ & $79.69 \%$ & $312 / 372$ & $83.87 \%$ & \\
\hline Fluid resuscitation* & $52 / 190$ & $27.37 \%$ & $66 / 109$ & $60.55 \%$ & \\
\hline $6 \mathrm{H}$ perfect care* & $41 / 140$ & $29.29 \%$ & $80 / 195$ & $41.03 \%$ & \\
\hline $6 \mathrm{H}$ lactate* & $84 / 132$ & $63.64 \%$ & $137 / 180$ & $76.11 \%$ & \\
\hline vasopressor* & $12 / 35$ & $34.29 \%$ & $23 / 48$ & $47.92 \%$ & \\
\hline Reassessment* & 0 & & $1 / 82$ & $1.22 \%$ & \\
\hline Total perfect care & $104 / 260$ & $40 \%$ & $187 / 372$ & $50.27 \%$ & \\
\hline
\end{tabular}

* the denominator based on the number of patients who met criteria for that intervention 
Our results showed that the usage of the sepsis order set increased significantly in the postintervention group $(p=0.001)$. We found that the postintervention group was 1.8 times more likely to use the order set than the preintervention group. The average time zero to antibiotic in postintervention group was 17.7 minutes lower than the preintervention group (Figure 12). However, the difference in time zero to antibiotic did not reach statistical significance. Furthermore, there was no significant difference in length of stay, survival, and total perfect care between the preintervention and postintervention groups.

We regrouped the whole population into the group that used sepsis order set (yes) and the group that did not use sepsis order set (no) and studied the association between using the sepsis order set and the primary outcomes (Figure 13). The average time zero to antibiotic in the order set use group was 54 minutes shorter than the group that did not use the order set, which was statistically significant with $p$-value $=0.001$. The average length of stay in the group that used the order set was 1.8 days shorter than the group that did not use the order set. The difference in length of stay showed statistically significant with the $p$-value of 0.002 . There was a nonsignificant trend towards improvement of survival in the group that used the order set.

Figure 13. The association between the usage of sepsis order set and the primary outcomes

\begin{tabular}{|c|c|c|c|}
\hline & \multicolumn{2}{|c|}{ Order set used } & \multirow[b]{2}{*}{$p$} \\
\hline & No $(n=255)$ & Yes $(n=377)$ & \\
\hline Time zero to antibiotic: average & 157 mins & 103 mins & $0.001^{\mathrm{a}}$ \\
\hline Inpatient length of stay: average & 7.28 days & 5.54 days & $0.002^{b}$ \\
\hline Survival rate at discharge & $229(89.8 \%)$ & $352(93.37 \%)$ & $0.109^{c}$ \\
\hline
\end{tabular}

We used the Chi-square statistic to determine the association between the order set use and total perfect care as shown in the $2 \times 2$ table (Figure 14). We found that the number of total 
perfect care increased significantly in the group that the order set was used with the $p$-value $<0.001$. Those who used the order set were 2.9 times more likely to achieve total perfect care than the group that did not use the order set.

Figure 14. The number of the usage of sepsis order set and the total perfect care

\begin{tabular}{ccc|c|c} 
& & \multicolumn{3}{c}{ Total perfect care } \\
\cline { 2 - 5 } & & No & Yes & Total \\
\hline \multirow{2}{*}{ Order set use } & No & 181 & 74 & 255 \\
\cline { 2 - 5 } & Yes & 160 & 217 & 377 \\
\hline Total & & 341 & 291 & 632 \\
\hline
\end{tabular}

We used the whole studied group of 632 patients and grouped into the group that achieved total perfect care (yes) and the group that did not achieve total perfect care (no). We assessed the association between the total perfect care and the primary outcomes (Figure 15). The group that achieved total perfect care had 102.4 minutes shorter average time zero to antibiotic $(p<0.001), 1.5$ days shorter average length of stay $(p=0.004)$, and better survival at discharge $(p<0.001,95 \%$ CI $0.02-0.206$, OR 0.064) than the group that did not achieve total perfect care.

Figure 15. The association between the total perfect care and the primary outcomes

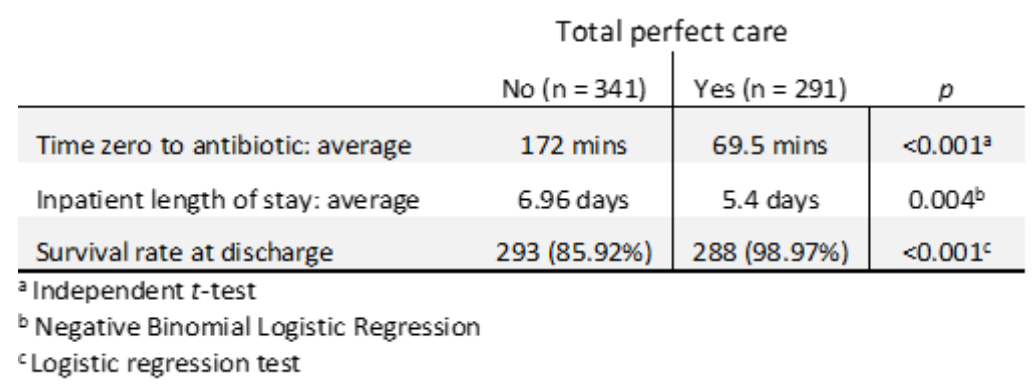




\section{Chapter 5 Discussion and analysis}

Our findings confirmed that the sepsis screening tool increased the usage of the order set, raised an awareness of the emergency department personnel and improved the adherence to the treatment guidelines by showing that the usage of sepsis order set significantly increased in the postintervention group $(p=0.001)$. The postintervention group was 1.8 times more likely to use the sepsis order set than the preintervention group, despite no direct link within the sepsis screening tool. Even though the sepsis screening tool did not improve primary outcomes, the postintervention group received antibiotics 17.7 minutes earlier than the preintervention group. There was no significant difference in time to antibiotic, length of stay, survival at discharge, and total perfect care when comparing before and after the launch of the sepsis screening tool. This study had some limitations that might impact the results. We did not adjust for clinical characteristics and severity of illness. Furthermore, the studied population might not large enough to detect the difference in the primary outcomes.

Our study found the improvement in outcome of treatment with the usage of sepsis order set. The utilization of sepsis order set streamlined and standardized the sepsis management which resulted in shorter time to antibiotic by 54 minutes $(p=0.001)$ and shorter length of stay by 1.8 days $(p=0.002)$. However, there was no significant difference in survival between the group that used the order set and the group that did not use the order set. Our results showed that there was a significant association between sepsis order set use and total perfect care $(p<0.001)$, which indicated that the order set use increased the compliance with SEP-1 measure.

Several studies reported the association between the compliance of standard treatment guidelines and mortality. Even with SSC compliance rates of less than $30 \%$, absolute reductions in mortality of $4-6 \%$ has been noted (Ferrer et al., 2008). National Quality Forum (NQF) stated 
that an absolute reduction in mortality over $20 \%$ was reported with compliance rated of $52 \%$ (NQF, 2012). Coba et al. (2011) reported that the mortality difference between patients with the completion of all SSC bundle elements compared to patients who had incomplete bundle elements was 14\%. SSC reported the significant association between compliance with SSC bundles and mortality in a 7.5-year study (Levy et al., 2015). The increased compliance with sepsis performance bundles associated with a $25 \%$ relative risk reduction in mortality rate (Levy et al., 2015). Every $10 \%$ increase in compliance and an additional quarter of participation in the SSC initiative was associated with a decrease in the odds ratio for hospital mortality (Levy et al., 2015).

However, the benefit of the compliance of the SEP-1 measure is unclear. The association between SEP-1 measure and mortality was evaluated in a multicenter retrospective study (Rhee et al., 2018). Rhee et al. (2018) reported that the crude mortality rates were higher in sepsis cases who failed to comply with CMS SEP-1 measure when comparing with sepsis cases who passed, but the difference was not significant after adjusting for clinical characteristics and severity of illness. Rhee's study concluded that detailed adjustment was necessary to properly interpret associations between SEP-1 compliance and mortality (Rhee et al., 2018)

Our results showed that the compliance of SEP-1, by achieving total perfect care, significantly improved all primary outcomes. The group that achieved total perfect care had significantly shortened the average time to antibiotics by 102.4 minutes $(p<0.001)$, shortened length of stay by 1.5 days $(p=0.004)$, and improved survival at discharge $(p<0.001,95 \%$ CI $0.02-0.206$, OR 0.064). Even though our results showed the compliance of SEP-1 strongly associated with primary outcomes, we need more studies to confirm these findings because we did not adjust for clinical characteristics and severity of illnesses. 
Our study had some limitations. The first limitation was the data that we used was extracted directly from the EMR, we lacked the details of clinical characteristics and severity of illness of the studied group. We are cautious about our results that we did not take clinical characteristics and severity of illness into account. The second limitation was the nature of a retrospective review at a single institution. The data was extracted directly from the EMR in which we had to rely on the records that might confound by the incompletion of the data. The third limitation of our study was that the number of studied populations might not be enough to detect significant differences between preintervention and postintervention groups. The last limitation was the unknown effect of partial treatments to the outcome of the study. Many patients underwent parts of the bundle elements but did not complete the required items in the bundle per SEP-1 measure requirement in which the benefit of partial treatments could become confounding factors of this study.

SSC study concluded that the performance metric could drive change in clinical behavior, improve quality of care, and may decrease mortality in patients with severe sepsis and septic shock (Levy et al., 2015). We believed that adherence to the performance metric of the quality measure would improve the outcomes of the treatment. Our study confirmed that adherence to the standard treatment guidelines improved the treatment outcomes. Even though the overall compliance of the SEP-1 measure in this study was 46\% (291/632), our study demonstrated the benefits of the sepsis screening tool, the benefits of sepsis order set, and the benefits of compliance of SEP-1 measure. In the future, we will aim to increase the use of sepsis order set, improve the alert system to responsible providers and link the sepsis order set directly at the sepsis screening tool. 


\section{Chapter 6 conclusion}

We concluded that the sepsis screening tool increased the usage of the sepsis order set and improved awareness of sepsis in the emergency triage. The group that used the sepsis order set had significantly shorter length of stay and shorter time to antibiotic. When the sepsis order set was used, the compliance with the SEP-1 measure increased. The group that met SEP-1 measure compliance significantly received antibiotics earlier, shorter stay as inpatient, and better survival. However, our study did not adjust for clinical characteristics and severity of illness. We need more investigations to confirm the association between compliance of SEP-1 measure and patient-related outcomes. 


\section{Bibliography}

Allison, M. G., \& Schenkel, S. M. (2018). SEP-1: A Sepsis Measure in Need of Resuscitation? Ann Emerg Med, 71(1), 18-20. doi:10.1016/j.annemergmed.2017.08.057

Amland, R. C., \& Hahn-Cover, K. E. (2016). Clinical Decision Support for Early Recognition of Sepsis. Am J Med Qual, 31(2), 103-110. doi:10.1177/1062860614557636

Amland, R. C., \& Hahn-Cover, K. E. (2019). Clinical Decision Support for Early Recognition of Sepsis(). Am J Med Qual, 34(5), 494-501. doi:10.1177/1062860619873225

Amland, R. C., Haley, J. M., \& Lyons, J. J. (2016). A Multidisciplinary Sepsis Program Enabled by a Two-Stage Clinical Decision Support System: Factors That Influence Patient Outcomes. Am J Med Qual, 31(6), 501-508. doi:10.1177/1062860615606801

Barbash, I. J., Rak, K. J., Kuza, C. C., \& Kahn, J. M. (2017). Hospital Perceptions of Medicare's Sepsis Quality Reporting Initiative. J Hosp Med, 12(12), 963-968. doi:10.12788/jhm.2929

Benthin, C., Pannu, S., Khan, A., \& Gong, M. (2016). The Nature and Variability of Automated Practice Alerts Derived from Electronic Health Records in a U.S. Nationwide Critical Care Research Network. Ann Am Thorac Soc, 13(10), 1784-1788. doi:10.1513/AnnalsATS.201603-172BC

Bhattacharjee, P., Edelson, D. P., \& Churpek, M. M. (2017). Identifying Patients With Sepsis on the Hospital Wards. Chest, 151(4), 898-907. doi:10.1016/j.chest.2016.06.020

Bone, R. C., Balk, R. A., Cerra, F. B., Dellinger, R. P., Fein, A. M., Knaus, W. A., ... Sibbald, W. J. (1992). Definitions for sepsis and organ failure and guidelines for the use of innovative therapies in sepsis. The ACCP/SCCM Consensus Conference Committee. 
American College of Chest Physicians/Society of Critical Care Medicine. Chest, 101(6), 1644-1655. doi:10.1378/chest.101.6.1644

Bresnick, J. (2017, December 12). Understanding the basics of clinical decision support systems. Retrieved from https://healthitanalytics.com/features/understanding-the-basics-of-

\section{clinical-decision-support-systems}

Brown, S. M., Jones, J., Kuttler, K. G., Keddington, R. K., Allen, T. L., \& Haug, P. (2016). Prospective evaluation of an automated method to identify patients with severe sepsis or septic shock in the emergency department. BMC Emerg Med, 16(1), 31.

doi:10.1186/s12873-016-0095-0

Campbell, R. (2013). The five "rights" of clinical decision support. J ahima, 84(10), 42-47; quiz 48.

Campbell, R. J. (2016, February). The Five Rights of Clinical Decision Support: CDS Tools Helpful for Meeting Meaningful Use. Retrieved from http://library.ahima.org/doc?oid=300027

De Backer, D., \& Dorman, T. (2017). Surviving Sepsis Guidelines: A Continuous Move Toward Better Care of Patients With Sepsis. Jama, 317(8), 807-808. doi:10.1001/jama.2017.0059

Dellinger, R. P., Levy, M. M., Rhodes, A., Annane, D., Gerlach, H., Opal, S. M., . . Moreno, R. (2013). Surviving Sepsis Campaign: international guidelines for management of severe sepsis and septic shock, 2012. Intensive Care Med, 39(2), 165-228. doi:10.1007/s00134$012-2769-8$

Dixon, B. E., Simonaitis, L., Goldberg, H. S., Paterno, M. D., Schaeffer, M., Hongsermeier, T., . . Middleton, B. (2013). A pilot study of distributed knowledge management and clinical 
decision support in the cloud. Artif Intell Med, 59(1), 45-53.

doi:10.1016/j.artmed.2013.03.004

Downing, N. L., Rolnick, J., Poole, S. F., Hall, E., Wessels, A. J., Heidenreich, P., \& Shieh, L. (2019). Electronic health record-based clinical decision support alert for severe sepsis: a randomised evaluation. BMJ Qual Saf. doi:10.1136/bmjqs-2018-008765

Esposito, A., Silverman, M. E., Diaz, F., Fiesseler, F., Magnes, G., \& Salo, D. (2018). Sepsis Core Measures - Are They Worth the Cost? J Emerg Med, 55(6), 751-757. doi:10.1016/j.jemermed.2018.07.033

Faust, J. S., \& Weingart, S. D. (2017). The Past, Present, and Future of the Centers for Medicare and Medicaid Services Quality Measure SEP-1: The Early Management Bundle for Severe Sepsis/Septic Shock. Emerg Med Clin North Am, 35(1), 219-231. doi:10.1016/j.emc.2016.09.006

Ferrer, R., Artigas, A., Levy, M. M., Blanco, J., Gonzalez-Diaz, G., Garnacho-Montero, J., . . . Edusepsis Study, G. (2008). Improvement in process of care and outcome after a multicenter severe sepsis educational program in Spain. Jama, 299(19), 2294-2303. doi:10.1001/jama.299.19.2294

Giannini, H. M., Ginestra, J. C., Chivers, C., Draugelis, M., Hanish, A., Schweickert, W. D., . . Umscheid, C. A. (2019). A Machine Learning Algorithm to Predict Severe Sepsis and Septic Shock: Development, Implementation, and Impact on Clinical Practice. Crit Care Med, 47(11), 1485-1492. doi:10.1097/ccm.0000000000003891

Gultepe, E., Green, J. P., Nguyen, H., Adams, J., Albertson, T., \& Tagkopoulos, I. (2014). From vital signs to clinical outcomes for patients with sepsis: a machine learning basis for a 
clinical decision support system. J Am Med Inform Assoc, 21(2), 315-325.

doi:10.1136/amiajnl-2013-001815

HealthIT.org. (n.d.). Clinical Decision Support. Retrieved from https://www.healthit.gov/topic/safety/clinical-decision-support

Horng, S., Sontag, D. A., Halpern, Y., Jernite, Y., Shapiro, N. I., \& Nathanson, L. A. (2017). Creating an automated trigger for sepsis clinical decision support at emergency department triage using machine learning. PLoS One, 12(4), e0174708. doi:10.1371/journal.pone.0174708

Kalantari, A., Mallemat, H., \& Weingart, S. D. (2017). Sepsis Definitions: The Search for Gold and What CMS Got Wrong. West J Emerg Med, 18(5), 951-956. doi:10.5811/westjem.2017.4.32795

Levy, M. M., Evans, L. E., \& Rhodes, A. (2018). The Surviving Sepsis Campaign Bundle: 2018 update. Intensive Care Med, 44(6), 925-928. doi:10.1007/s00134-018-5085-0

Levy, M. M., Fink, M. P., Marshall, J. C., Abraham, E., Angus, D., Cook, D., . . Ramsay, G. (2003). 2001 SCCM/ESICM/ACCP/ATS/SIS International Sepsis Definitions Conference. Intensive Care Med, 29(4), 530-538. doi:10.1007/s00134-003-1662-x

Levy, M. M., Gesten, F. C., Phillips, G. S., Terry, K. M., Seymour, C. W., Prescott, H. C., . . Lemeshow, S. (2018). Mortality Changes Associated with Mandated Public Reporting for Sepsis. The Results of the New York State Initiative. Am J Respir Crit Care Med, 198(11), 1406-1412. doi:10.1164/rccm.201712-2545OC

Levy, M. M., Rhodes, A., Phillips, G. S., Townsend, S. R., Schorr, C. A., Beale, R., .. . Dellinger, R. P. (2015). Surviving Sepsis Campaign: association between performance 
metrics and outcomes in a 7.5-year study. Crit Care Med, 43(1), 3-12. doi:10.1097/ccm.0000000000000723

Li, L., Rathnayake, K., Green, M., Fullick, M., Shetty, A., Walter, S., . . Westbrook, J. I. (2019). Improving the Performance of Clinical Decision Support for Early Detection of Sepsis: A Retrospective Observational Cohort Study. Stud Health Technol Inform, 264, 679-683. doi:10.3233/shti190309

Makam, A. N., Nguyen, O. K., \& Auerbach, A. D. (2015). Diagnostic accuracy and effectiveness of automated electronic sepsis alert systems: A systematic review. J Hosp Med, 10(6), 396-402. doi:10.1002/jhm.2347

Manaktala, S., \& Claypool, S. R. (2017). Evaluating the impact of a computerized surveillance algorithm and decision support system on sepsis mortality. J Am Med Inform Assoc, 24(1), 88-95. doi:10.1093/jamia/ocw056

Mouncey, P. R., Osborn, T. M., Power, G. S., Harrison, D. A., Sadique, M. Z., Grieve, R. D., . . Rowan, K. M. (2015). Protocolised Management In Sepsis (ProMISe): a multicentre randomised controlled trial of the clinical effectiveness and cost-effectiveness of early, goal-directed, protocolised resuscitation for emerging septic shock. Health Technol Assess, 19(97), i-xxv, 1-150. doi:10.3310/hta19970

National Quality Forum. (2012, October 5). Severe Sepsis and Septic Shock: Management Bundle. Retrieved from https://www.google.com/url?sa=t\&rct=j\&q=\&esrc=s\&source=web\&cd=1\&cad=rja\&uact $=8 \&$ ved $=2$ ahUKEwig $4 \mathrm{~K}-4-$ vTlAhVIRqwKHYVMBZ0QFjAAegQIARAC\&url=https\%3A\%2F\%2Fwww.qualityforu m.org\%2FProjects\%2Fi- 
$\underline{\text { m\%2FInfectious_Disease_Endorsement_Maintenance_2012\%2F0500.aspx\&usg=AOvV }}$ $\underline{\text { aw348AX9kQGABrPB7K8vp5Jn }}$

Nemati, S., Holder, A., Razmi, F., Stanley, M. D., Clifford, G. D., \& Buchman, T. G. (2018). An Interpretable Machine Learning Model for Accurate Prediction of Sepsis in the ICU. Crit Care Med, 46(4), 547-553. doi:10.1097/ccm.00000000000002936

Nesseler, N., Defontaine, A., Launey, Y., Morcet, J., Malledant, Y., \& Seguin, P. (2013). Longterm mortality and quality of life after septic shock: a follow-up observational study. Intensive Care Med, 39(5), 881-888. doi:10.1007/s00134-013-2815-1

Nguyen, H. B., Jaehne, A. K., Jayaprakash, N., Semler, M. W., Hegab, S., Yataco, A. C., . . Rivers, E. P. (2016). Early goal-directed therapy in severe sepsis and septic shock: insights and comparisons to ProCESS, ProMISe, and ARISE. Crit Care, 20(1), 160. doi:10.1186/s13054-016-1288-3

Nguyen, S. Q., Mwakalindile, E., Booth, J. S., Hogan, V., Morgan, J., Prickett, C. T., . . Wang, H. E. (2014). Automated electronic medical record sepsis detection in the emergency department. PeerJ, 2, e343. doi:10.7717/peerj.343

Novosad, S. A., Sapiano, M. R., Grigg, C., Lake, J., Robyn, M., Dumyati, G., . . Epstein, L. (2016). Vital Signs: Epidemiology of Sepsis: Prevalence of Health Care Factors and Opportunities for Prevention. MMWR Morb Mortal Wkly Rep, 65(33), 864-869. doi:10.15585/mmwr.mm6533e1

Osheroff, J.A., Teich, J.M., Levick, D., Saldana, L., Velasco, F.T., Sittig, D.F., Rogers, K.M., \& Jenders, R.A. (2012). Improving Outcomes with Clinical Decision Support: An implementer's guide (2 $2^{\text {nd }}$. ed.). Boca Raton, FL: CRC Press. 
Pepper, D. J., Natanson, C., \& Eichacker, P. Q. (2018). Evidence Underpinning the Centers for Medicare \& Medicaid Services' Severe Sepsis and Septic Shock Management Bundle (SEP-1). Ann Intern Med, 168(8), 610-612. doi:10.7326/118-0140

Rhee, C., Filbin, M. R., Massaro, A. F., Bulger, A. L., McEachern, D., Tobin, K. A., . . . Klompas, M. (2018). Compliance With the National SEP-1 Quality Measure and Association With Sepsis Outcomes: A Multicenter Retrospective Cohort Study. Crit Care Med, 46(10), 1585-1591. doi:10.1097/ccm.0000000000003261

Rhodes, A., Evans, L. E., Alhazzani, W., Levy, M. M., Antonelli, M., Ferrer, R., ... Dellinger, R. P. (2017). Surviving Sepsis Campaign: International Guidelines for Management of Sepsis and Septic Shock: 2016. Intensive Care Med, 43(3), 304-377. doi:10.1007/s00134$017-4683-6$

Rivers, E., Nguyen, B., Havstad, S., Ressler, J., Muzzin, A., Knoblich, B., . . . Early GoalDirected Therapy Collaborative, G. (2001). Early goal-directed therapy in the treatment of severe sepsis and septic shock. N Engl J Med, 345(19), 1368-1377. doi:10.1056/NEJMoa010307

Rolnick, J., Downing, N. L., Shepard, J., Chu, W., Tam, J., Wessels, A., . . Shieh, L. (2016). Validation of Test Performance and Clinical Time Zero for an Electronic Health Record Embedded Severe Sepsis Alert. Appl Clin Inform, 7(2), 560-572. doi:10.4338/aci-201511-ra-0159

Rowan, K. M., Angus, D. C., Bailey, M., Barnato, A. E., Bellomo, R., Canter, R. R., .. Yealy, D. M. (2017). Early, Goal-Directed Therapy for Septic Shock - A Patient-Level MetaAnalysis. N Engl J Med, 376(23), 2223-2234. doi:10.1056/NEJMoa1701380 
Rusconi, A. M., Bossi, I., Lampard, J. G., Szava-Kovats, M., Bellone, A., \& Lang, E. (2015). Early goal-directed therapy vs usual care in the treatment of severe sepsis and septic shock: a systematic review and meta-analysis. Intern Emerg Med, 10(6), 731-743. doi:10.1007/s11739-015-1248-y

Semler, M. W., Weavind, L., Hooper, M. H., Rice, T. W., Gowda, S. S., Nadas, A., .. . Wheeler, A. P. (2015). An Electronic Tool for the Evaluation and Treatment of Sepsis in the ICU: A Randomized Controlled Trial. Crit Care Med, 43(8), 1595-1602. doi:10.1097/ccm.0000000000001020

Seymour, C. W., Gesten, F., Prescott, H. C., Friedrich, M. E., Iwashyna, T. J., Phillips, G. S., . . Levy, M. M. (2017). Time to Treatment and Mortality during Mandated Emergency Care for Sepsis. N Engl J Med, 376(23), 2235-2244. doi:10.1056/NEJMoa1703058

Seymour, C. W. \& Weirsinga, W. J. (2018). Handbook of Sepsis. Cham, Switzerland: Springer International Publishing AG.

Shimabukuro, D. W., Barton, C. W., Feldman, M. D., Mataraso, S. J., \& Das, R. (2017). Effect of a machine learning-based severe sepsis prediction algorithm on patient survival and hospital length of stay: a randomised clinical trial. BMJ Open Respir Res, 4(1), e000234. doi:10.1136/bmjresp-2017-000234

Singer, M., Deutschman, C. S., Seymour, C. W., Shankar-Hari, M., Annane, D., Bauer, M., .. . Angus, D. C. (2016). The Third International Consensus Definitions for Sepsis and Septic Shock (Sepsis-3). Jama, 315(8), 801-810. doi:10.1001/jama.2016.0287

Taylor, R. A., Pare, J. R., Venkatesh, A. K., Mowafi, H., Melnick, E. R., Fleischman, W., \& Hall, M. K. (2016). Prediction of In-hospital Mortality in Emergency Department 
Patients With Sepsis: A Local Big Data-Driven, Machine Learning Approach. Acad Emerg Med, 23(3), 269-278. doi:10.1111/acem.12876

Torio, C. M., \& Moore, B. J. (2016). National Inpatient Hospital Costs: The Most Expensive Conditions by Payer, 2013: Statistical Brief \#204. In Healthcare Cost and Utilization Project (HCUP) Statistical Briefs. Rockville (MD): Agency for Healthcare Research and Quality (US).

Venkatesh, A. K., Slesinger, T., Whittle, J., Osborn, T., Aaronson, E., Rothenberg, C., .. . Schuur, J. D. (2018). Preliminary Performance on the New CMS Sepsis-1 National Quality Measure: Early Insights From the Emergency Quality Network (E-QUAL). Ann Emerg Med, 71(1), 10-15.e11. doi:10.1016/j.annemergmed.2017.06.032

Wiersinga, W.J. \& Seymour, C. W. (2018). Handbook of Sepsis. Cham, Switzerland. Springer International Publishing AG.

Yealy, D. M., Kellum, J. A., Huang, D. T., Barnato, A. E., Weissfeld, L. A., Pike, F., ... Angus, D. C. (2014). A randomized trial of protocol-based care for early septic shock. $N$ Engl J Med, 370(18), 1683-1693. doi:10.1056/NEJMoa1401602 